\title{
HandsOn e Compsi: Parceria em Projetos de Realidade Virtual para Indústria, Comércio e Saúde
}

\author{
Allan Oliveira ${ }^{12}$, Pedro Lopes ${ }^{13}$, Leonardo Santos ${ }^{1}$, Allan Cruz ${ }^{12}$, Daniel Silva ${ }^{12}$, Luciamare Martins ${ }^{3}$ \\ ${ }^{1}$ HandsOn Soluções Tecnológicas - https://handsonmedical.com/ \\ ${ }^{2}$ Laboratório Compsi, Univem - https://compsi.univem.edu.br \\ 3Departamento de Parasitologia da Faculdade de Medicina de Marília (FAMEMA) - http://www.famema.br/ \\ allancaixeta@gmail.com, pedro.henrique.p.l@hotmail.com, leoademir0@gmail.com, allan.morishigue@hotmail.com, \\ dmoyasilva98@gmail.com, luciamarepam@gmail.com
}

\begin{abstract}
Resumo: A empresa HandsOn, em parceria com o laboratório de pesquisa Compsi do Centro universitário Eurípides de Marília Univem, foi criada para atender demandas de sistemas de interfaces computacionais. Ela originou para desenvolver um sistema de apoio a interação médico-paciente, mostrando de forma visual doenças e tratamentos. Em sequência ela se envolveu na área de construção civil, criando um sistema para lojas de construção usarem com seus clientes para montarem sua casa na Realidade Virtual. E por fim ela desenvolveu um sistema de treinamento para operadores de fábrica, que permite rápida construção de procedimentos de treinamentos por gestores. Como principal contribuição, a empresa publicou o framework de código aberto Project Team, que acelera o ciclo de desenvolvimento da Realidade Virtual.
\end{abstract}

Palavras-chave: realidade virtual; treinamento; interação médico-paciente.

\section{Introdução}

A empresa HandsOn nasceu com o propósito de produzir tecnologias que sejam de fácil acesso e interação para seus usuários.

Desde a sua fundação ela tem uma forte relação com o laboratório de pesquisa Compsi do Centro universitário Eurípides de Marília - Univem, localizado na cidade de Marília.

A empresa atua produzindo softwares de Realidade Virtual (RV), Realidade Aumentada (RA) e Computação Gráfica de forma geral, principalmente na indústria, comércio e saúde.

\section{Principais Projetos}

A seguir serão apresentados os principais projetos da HandsOn em parceria com o laboratório de pesquisa Compsi da Univem de Marília.

\subsection{Treinamento de operadores de indústria alimentícia}

Com a presença cada vez mais frequente da tecnologia na indústria e seu aumento aplicado em processos internos, tecnologias como a RV e RA tem se tornado cada vez mais presentes $[1,2,3,4]$, sendo utilizada para treinamentos [2], monitoramentos [3] e simulações [4].

Devido a isso, a informatização - sendo em partes automatização e virtualização - de processos tem se tornado cada vez mais habitual, dando origem ao termo conhecido como indústria 4.0 [5].

Nesse sentido, o sistema para treinamento de operadores em ambiente virtual foi desenvolvido por uma demanda de uma fábrica na área de alimentos da cidade de Marília.

Os operadores da fábrica passam atualmente por um processo de treinamento teórico, seguido de um treinamento in loco. Porém cada linha de produção possui equipamentos diferentes, produz produtos diferentes, e, portanto, necessita de habilidades e conhecimentos diferentes.

Mesmo considerando operadores já treinados, é necessário fazer a reciclagem do treinamento periodicamente, garantindo que o procedimento continua sendo seguido e que o operador não adquira vícios (desvio) na sua execução do procedimento.

O sistema visa contribuir na automatização, facilitação, melhora na interatividade e feedback sobre o treinamento do operador em seu procedimento de fabricação de produtos.

Além disso, devido ao sistema ser totalmente digital, sempre que o treinamento for aplicado, não há a necessidade de interagir com os equipamentos do meio físico da fábrica, possibilitando fazer o treinamento quando quiser sem que prejudique a produção dos produtos.

Visando essas dificuldades, foi construído um sistema de treinamento em ambiente virtual. Esse sistema pode ser usado nas plataformas PC e RV. Todas as ações de interação da RV foram abstraídas no PC para serem "point and click", permitindo uma experiência similar para operadores que sintam desorientação na RV.

Para garantir a escalabilidade do sistema, foi desenvolvido uma ferramenta de modelagem de treinamentos, usada inicialmente por designers e futuramente pelos gestores da fábrica aderindo ao EndUser Development [6].

Essa ferramenta permite modelar treinamentos no ambiente virtual através da construção de uma máquina de estados das ações que o usuário deve fazer durante o treinamento. Conforme mostrado na Figura 1, cada estado representa uma etapa do treinamento com uma ação (um verbo) e um objeto.

Outro recurso do sistema é a possibilidade de criar "fases" com diferentes níveis de dificuldade para cada procedimento. Esse recurso proporciona variabilidade, facilitando na repetição necessária para transformar a habilidade do treinamento em conhecimento.

Com o recurso de fases temos a pontuação e placar. Cada fase de um procedimento realizado gera uma nota para o usuário, calculada com base no tempo de execução e quantidade de erros cometidos. Essas notas 
servem primariamente como avaliação de competência, como trava para fases mais avançadas, e também são expostas em um placar para estimular competição entre colaboradores.

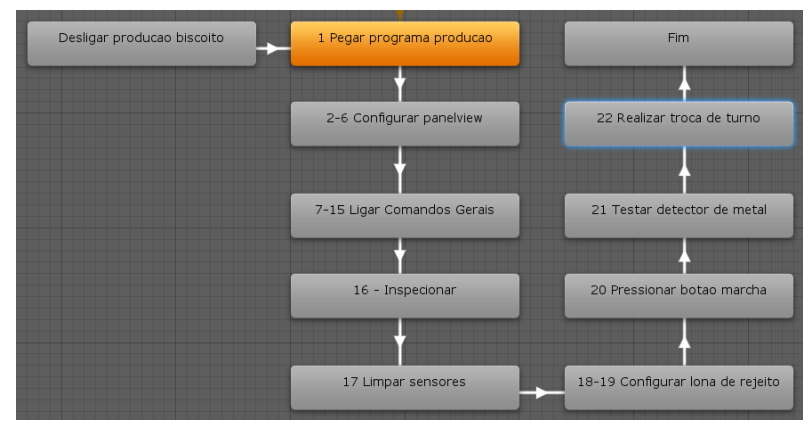

Figura 1. Máquina de estados definindo etapas do treinamento.

Outro recurso do sistema é um NPC colaborador. O NPC pode receber um comando para cada estado do procedimento, de forma que ele simula um colaborador real fazendo um procedimento com várias pessoas. Ele se comunica com o usuário através de um conjunto de gestos, como na Figura 2 em que ele está fazendo um gesto de “jóia”.

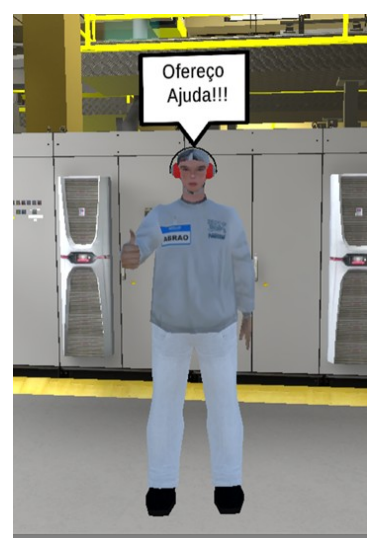

Figura 2. NPC colaborador fazendo gesto de prosseguir (jóia) para o usuário.

Um dos possíveis erros que o usuário pode cometer é justamente fazer uma ação antes do NPC estar pronto. O NPC também serve como um tutorial para ajudar usuários que esqueceram do procedimento.

Por fim, o sistema também tem um dashboard gráfico para gestores acompanharem o resultado dos treinamentos e avanço da equipe operacional, assim como identificar hotspots de dificuldade dos colaboradores para remodelar o treinamento.

\subsection{Sistema de apoio a interação médico-paciente}

$\mathrm{Na}$ área da saúde temos um problema que ocorre com muita frequência, que é a não-adesão aos tratamentos das doenças. Essa não-adesão é um dos principais motivos do insucesso a terapêutica/terapia [7].

Pacientes adoecidos necessitam entender a sua condição e sua terapêutica, sendo de fundamental importância a interação comunicativa entre o médico e o paciente [8]. Porém, a dificuldade no não entendimento do paciente pode levá-lo a se desviar do tratamento ou não entender a importância do tratamento e a gravidade de sua situação. Nesse caso temos um problema educacional, que diversos projetos $[9,10]$ provam que o auxílio de imagens e vídeos melhoram a absorção do conhecimento.

Com essa finalidade nasceu o HandsOn Medical Support, um sistema para apoiar a interação médicopaciente. Se trata de um sistema web interativo que permite visualizar o corpo humano em 3D (Figura 3). Atualmente o sistema possui somente a especialidade da oftalmologia mapeada e está aberto a acesso no link: https://handsonmedical.com/oftalmo/
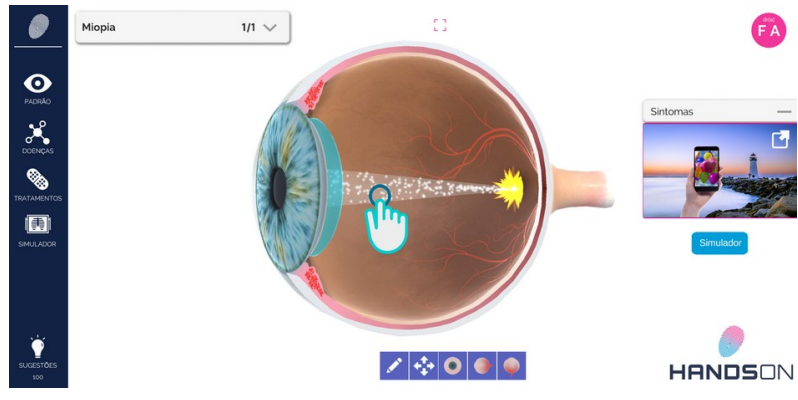

Figura 3. Sistema de apoio a interação médico-paciente da HandsOn.

Esse sistema apresenta como a enfermidade altera o paciente (como por exemplo, miopia), como ela pode progredir e ficar cada vez pior, além do resultado após o tratamento.

Ele mostra patologias e tratamentos através de animações 3D ou interações do usuário. Por exemplo, doenças mais complexas como o glaucoma são mostradas em animações, enquanto doenças mais simples como a miopia permitem que o usuário clique e interaja com o olho humano para ver seu efeito.

O objetivo é auxiliar na interação médico-paciente, apoiando o médico no consultório em sua explicação ao paciente, e aumentando a adesão ao tratamento. Para isso ele também permite que o médico desenhe na tela sobre as doenças, usando o mouse ou monitor que suporte toques.

Além da simulação 3D de doenças e tratamentos, o sistema tem um simulador de efeitos na visão humana, que permite simular algumas das principais doenças oculares. A Figura 4 mostra no simulador a visão em um estado avançado de Degeneração Macular.

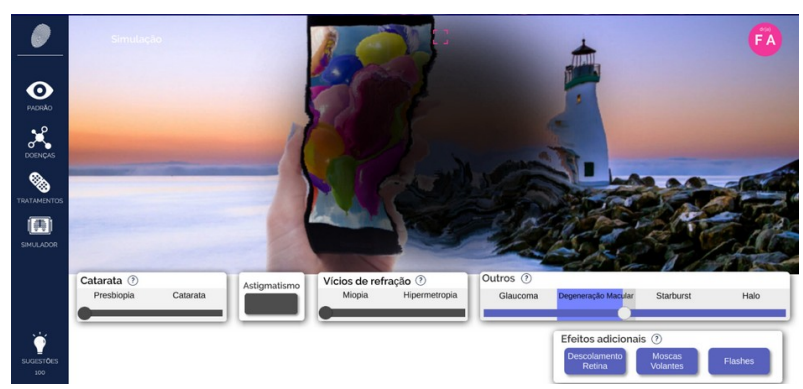

Figura 4. Simulador de visão mostrando estágio avançado de Degeneração Macular. 


\subsection{CivilRV}

O sistema CivilRV foi desenvolvido para atender uma demanda de lojas de construção civil. Clientes dessas lojas tem dificuldade de escolha de produtos e de imaginar como a combinação de produtos que eles desejam ficam em um ambiente real. O sistema foi criado justamente para permitir configurar, em um ambiente virtual, cada produto que o cliente deseja comprar.

O sistema, que funciona em RV ou PC, permite ao cliente configurar o seu cômodo com os produtos da loja. Por exemplo na Figura 5 o usuário na RV está selecionando um modelo de vaso sanitário.

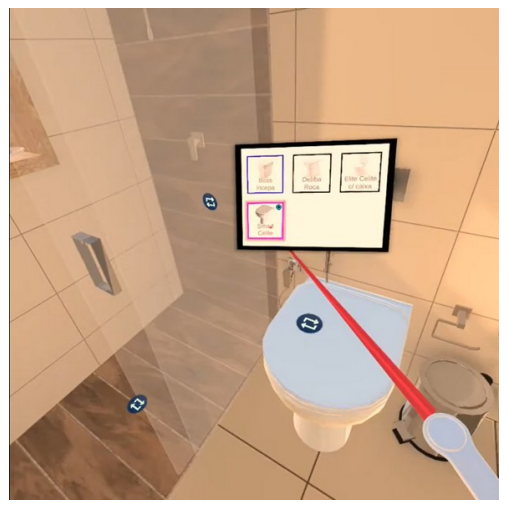

Figura 5. Seleção do modelo de vaso sanitário na Realidade Virtual por um usuário.

\section{Contribuições da HandsOn e Compsi}

Cada projeto tem suas contribuições sociais, ao facilitar e agilizar processos existentes, ajudando o operador de fábrica, o paciente e o comprador.

O sistema de treinamento gera alguns benefícios sociais como possibilidade de geração de um vídeo de treinamento e vídeo de marketing institucional, limitação na exposição de operadores não treinados a linha e até facilidade em aplicar reciclagens.

O sistema de interação médico-paciente também gera contribuições sociais, visando que a atenção visual contribui com redução de esforço cognitivo [11], além do auxílio ao entendimento, diminuindo a taxa de abandono a terapêutica.

O sistema CivilRV ajuda compradores em suas decisões, para que consigam visualizar o resultado das suas compras antes da aplicação dos produtos.

Em termos de contribuição científica, o destaque é o sistema de interação médico-paciente, que envolveu diversos alunos de Iniciação Científica, e está produzindo no momento um trabalho de mestrado no qual é realizado a comparação de seis softwares para resultar em qual tecnologia é mais efetiva na interação médico-paciente, para por fim, ter um framework que inclui as melhores tecnologias como resultado.

Em termos de contribuição tecnológica, os projetos produziram o framework de código aberto no topo do engine Unity para desenvolvimento de Realidade Virtual, denominado Project Team: https://bitbucket.org/ allancaixeta/projectteamframework

\subsection{Framework de RV Project Team}

O framework Project Team é voltado para a engine Unity e para os equipamentos Oculus Go e Oculus Quest. Ele tem dois objetivos, acelerar o desenvolvimento de sistemas de RV e permitir desenvolvimento multiplataforma com dois tipos de interação: point and click e 6DoF.

$\mathrm{O}$ desenvolvimento multiplataforma permite criar sistemas que são de RV e usam a interação 6DoF ou PC e usam a interação point and click. Dessa forma o mesmo sistema funciona de forma similar em duas plataformas diferentes, requisito não funcional importante pois nem todos os usuários se adaptam bem a RV. O framework também promove a não necessidade de ter um capacete (HMD) de RV para realizar testes iniciais.

O framework possui três recursos principais: objetos prontos (prefabs); scripts de interações em objetos (Selectors); scripts gerais.

Os objetos prontos são, por exemplo, câmera e personagem prontos para funcionar na RV e PC, com funcionalidades de locomoção, mira e interação já preparadas. Assim como canvas de interfaces prontas para serem usados na tela (modelo HUD) no PC ou fixo nas mãos do avatar na RV.

Em termos de scripts de interação, chamados de Selectors no framework, eles são seletores responsáveis por realizar diversas interações em objetos $3 d$ tendo alguma alteração em cena como resultado.

Por fim, o framework tem alguns scripts para uso geral no desenvolvimento de sistemas de RV, como scripts para gerenciar as mãos e ações de pegar e soltar, scripts para realizar ações a partir de botões do controle, e outros de uso geral.

\section{Conclusões}

Há um grau de dificuldade elevado para levantar, avaliar e aplicar os procedimentos que são aplicados na fábrica, pois além da necessidade de fidelizar o ambiente o mais próximo possível da realidade, também é necessário abstrair e inserir feedbacks instantâneos ao colaborador, para que o mesmo possa ver sua evolução e entender a necessidade do treinamento em um ambiente virtualizado.

Assim como no sistema da interação médicopaciente, é necessário que o paciente possa ver o mais próximo possível do mundo real a sua atual situação, trazendo um melhor entendimento.

Por fim, com a possibilidade de visualizar da forma mais palpável possível de como ficarão os cômodos da casa, o sistema Civil RV traz a imersão necessária para que o cliente possa se sentir dentro do seu próprio cômodo mobiliado, trazendo uma maior satisfação e feedback de como ficará futuramente.

\section{Bibliografia}

[1] Berg, L.; Vance, J. (2017) Industry use of virtual reality in product design and manufacturing: a survey. Virtual Reality 21(1): 1-17. DOI: 10.1007/s10055-016-0293-9 
[2] Goulding, J.; Nadim, W.; Petridis, P.; Alshawi, M. (2012) Construction industry offsite production: a virtual reality interactive training environment prototype. Advanced Engineering Informatics 26(1): 103-116. DOI: 10.1016/j.aei.2011.09.004

[3] Alam, M. F.; Katsikas, S.; Beltramello, O.; Hadjiefthymiades, S. (2017) Augmented and virtual reality based monitoring and safety system: A prototype IoT platform. Journal of Network and Computer Applications 89(1): 109-119. DOI: 10.1016/j.jnca.2017.03.022

[4] Grantcharov, T. P.; Rosenberg, J.; Pahle, E.; Fench-Jensen, P. (2001) Virtual reality computer simulation. Surgical Endoscopy 15(3): 242-244. DOI: 10.1007/s004640090008

[5] Lasi, H.; Fettke, P.; Kemper, H.-G.; Feld, T.; Hoffmann, M. (2014) Industry 4.0. Business \& Information Systems Engineering 6(4): 239-242. DOI: 10.1007/s12599-0140334-4

[6] Lieberman H.; Paternò F.; Klann M.; Wulf V. (2006) Enduser development: An emerging paradigm. In: Lieberman H., Paternò F., Wulf V. (eds) End User Development. Human-Computer Interaction Series, vol 9. Springer. DOI: $\underline{10.1007 / 1-4020-5386-X \quad 1}$
[7] Gallagher, E.J., Viscoli, C.M., \& Horwitz, R.I. (1993) The relationship of treatment adherence to the risk of death after myocardial infarction in women. The Journal of the American Medical Association 270(6): 742-744. DOI: 10.1001/jama.1993.03510060088038

[8] Schneider, P.B. (1974) Psicologia aplicada a la practica médica. Buenos Aires, Editorial Paidos.

[9] Belmiro, C.A. (2000) A imagem e suas formas de visualidade nos livros didáticos de Português. Educação \& Sociedade 21(72): 11-31. DOI: 10.1590/S010173302000000300002

[10] Bates, A.W.; Poole, G. (2003) Effective Teaching with Technology in Higher Education: Foundations for Success: 1. ed. Jossey-Bass. DOI: 10.1353/pla.2004.0034

[11] Silva, L.; Nakamura, R.; Nunes, F. (2019) Visualização 3D interativa de dados médicos temporais baseada em modelo de atenção visual. In: Anais Estendidos do XXI Simpósio de Realidade Virtual e Aumentada, p. 17-18. SBC. DOI: $10.5753 /$ svr estendido.2019.8456 